\title{
Intertwiners in Orbifold Conformal Field Theories
}

\author{
P.S. Montague \\ Department of Mathematics \\ University of Adelaide \\ Adelaide \\ South Australia 5005 \\ Australia
}

July 30, 2013

\begin{abstract}
Following on from earlier work relating modules of meromorphic bosonic conformal field theories to states representing solutions of certain simple equations inside the theories, we show, in the context of orbifold theories, that the intertwiners between twisted sectors are unique and described explicitly in terms of the states corresponding to the relevant modules. No explicit knowledge of the structure of the twisted sectors is required. Further, we propose a general set of sufficiency conditions, illustrated in the context of a third order no-fixed-point twist of a lattice theory, for verifying consistency of arbitrary orbifold models in terms of the states representing the twisted sectors.
\end{abstract}

\section{Introduction}

In previous papers [12, 10] we have introduced a scheme whereby to each representation of a hermitian bosonic meromorphic conformal field theory (CFT) we assign a certain state, generically denoted by $P$. In [12] a set of equations was derived which $P$ is required to satisfy, while in [10] the general solution to these equations was found, and in the particular example of $\mathbf{Z}_{2}$-twisted orbifolds of the lattice theories this was found to be sufficient to prove the anticipated "uniqueness of the twisted representation". It is conjectured in general that all solutions to these equations correspond to representations (there is a constructive procedure given in [12], though some technicalities remain to be demonstrated in general), i.e. that the correspondence between representations and solutions of certain equations inside the CFT is one-to-one. As an immediate application, we see that the calculations involved in verifying that a $\mathbf{Z}_{2}$-orbifold of a CFT is itself consistent as a CFT may be reformulated entirely in terms of the Hilbert space and vertex operators of the original CFT (specifically its $\mathbf{Z}_{2}$-invariant projection), and no explicit realisation of the twisted sector is necessary, c.f. [8, 园, 回.

For orbifolds of order higher than 2, we have also to consider vertex operators intertwining the various twisted sectors when discussing the orbifold theory. In this paper, we demonstrate that similar results hold true, i.e. that we may completely define the structure of the orbifold 
CFT with reference only to states and vertex operators inside the (twist-invariant projection of the) original CFT. We show that, given two (twisted) representations corresponding to states $P_{1}$ and $P_{2}$ of a CFT there is at most one intertwiner corresponding to the action of one on the other, and further we demonstrate how to calculate the appropriate matrix elements in terms of $P_{1}$ and $P_{2}$. Thus, given that the above mentioned conjecture of a one-to-one correspondence is true, we can verify completely all of the locality relations necessary for the orbifold CFT to be consistent entirely in terms of the $P$ 's corresponding to the twisted sectors and the structure of the original CFT. We stress again that no explicit knowledge of the twisted sectors is necessary (though it is all encoded in the $P$ 's) and the method is thus of great import when a geometric interpretation of the orbifold is lacking and it is therefore unclear how to begin the construction explicitly. Applications to physically realistic string models and also to completion of the classification of central charge 24 self-dual conformal field theories, by explicitly constructing the possible theories identified by Schellekens [13, 14] as orbifolds of the existing theories, are immediately apparent.

As an illustrative example, we consider the no-fixed-point $\mathbf{Z}_{3}$-orbifold of a lattice theory $\mathcal{H}(\Lambda)$ discussed in [11]. The complicated (and incomplete) calculations therein necessary to verify consistency of the orbifold theory may be recast entirely in terms of two states $P_{1}$ and $P_{2}$ in $\mathcal{H}(\Lambda)$ corresponding to the two twisted sectors (we have two non-trivial conjugacy classes in the $\mathbf{Z}_{3}$ group of automorphisms $\langle\theta\rangle$ ). In [11], an ansatz was made for the form of the intertwiner between the two twisted sectors and the coefficients explicitly evaluated (recovering results of Gato in [6]). As we now see, this is not necessary - no assumptions need be made. Also, in this case, an explicit realisation of the twisted sectors is known, and we do not even require our conjecture about the one-to-one correspondence between solutions $P$ of the equations of [12, 10] and representations to hold true. Consistency of the $\mathbf{Z}_{3}$-orbifold may hence be verified, and further we demonstrate that much of the calculation

of [11] is unnecessary due to a relation between $P_{1}$ and $P_{2}$ which generalises to arbitrary orbifold theories.

The layout of the paper is as follows.

In section 2 we summarise and generalise to distinct, non-quasi-primary and non-real representation states the results of [12] and [10].

In section 3, we extend this work to consider intertwiners between distinct representations, while in section 0 we illustrate our discussion first with a trivial application to the Heisenberg algebra before considering the more sophisticated example of the no-fixed-point $\mathbf{Z}_{3}$-orbifold of $\mathcal{H}(\Lambda)$.

In the appendix, we demonstrate the rather surprising result that an apparently natural (and considerably more amenable to calculation) ansatz for the form of the twisted sectortwisted sector intertwiner necessarily fails in general.

\section{Construction of the state $P$}

Let us first establish our notation. We define a conformal field theory (strictly a bosonic, hermitian, meromorphic conformal field theory) to consist of a Hilbert space $\mathcal{H}$, two fixed states $|0\rangle, \psi_{L} \in \mathcal{H}$, and a set $\mathcal{V}$ of "vertex operators", i.e. linear operators $V(\psi, z): \mathcal{H} \rightarrow \mathcal{H}$, $\psi \in \mathcal{H}$ parameterized by a complex parameter $z$ such that $V\left(\psi_{1}, z_{1}\right) V\left(\psi_{2}, z_{2}\right) \cdots$ makes sense for $\left|z_{1}\right|>\left|z_{2}\right|>\cdots$,

$$
V(\psi, z)|0\rangle=e^{z L_{-1}} \psi
$$




$$
V(\psi, z) V(\phi, w)=V(\phi, w) V(\psi, z)
$$

(the so-called "locality" relation - it holds in the sense that appropriate analytic continuations of matrix elements of either side agree) and

$$
V\left(\psi_{L}, z\right) \equiv \sum_{n \in \mathbf{Z}} L_{n} z^{-n-2},
$$

where

$$
\left[L_{m}, L_{n}\right]=(m-n) L_{m+n}+\frac{c}{12} m\left(m^{2}-1\right) \delta_{m,-n},
$$

(the constant $c$ is the "central charge" of the theory), with $L_{n}^{\dagger}=L_{-n}$. See [2] for a full discussion of this definition (as well as the more technical axioms omitted here). We will use the result

$$
\left[L_{-1}, V(\psi, z)\right]=\frac{d}{d z} V(\psi, z) .
$$

We define a representation (which we will take to be real and hermitian - see [2]) of this theory to consist of a Hilbert space $\mathcal{K}$ and a set of linear (vertex) operators $U(\psi, z): \mathcal{K} \rightarrow \mathcal{K}$, $\psi \in \mathcal{H}$, such that "duality" holds, i.e.

$$
U(\psi, z) U(\phi, w)=U(V(\psi, z-w) \phi, w),
$$

and also $U(|0\rangle) \equiv 1$. Note that a relation identical to (6) is satisfied by the $V$ 's as a consequence of the above axioms [7, 2]. This representation is said to be meromorphic if matrix elements of the $U$ 's are meromorphic functions of the complex arguments.

We also define vertex operators $W$ and the conjugate $\bar{W}$ by

$$
W(\chi, z) \psi=e^{z L_{-1}} U(\psi,-z) \chi
$$

and

$$
\bar{W}(\chi, z)=W\left(e^{z^{*} L_{1}} z^{*-2 L_{0}} \bar{\chi}, \frac{1}{z^{*}}\right)^{\dagger}
$$

where $\chi \mapsto \bar{\chi}$ is a certain antilinear involution (see [2] for details). [Note that, in general, $\bar{\chi}$ may lie in a distinct representation to $\chi$. In particular, in the case of the twisted representations which we will discuss below, if $\chi$ lies in a $g$-twisted representation than its conjugate $\bar{\chi}$ lies in a $g^{-1}$-twisted representation.] There is a similar involution on $\mathcal{H}$, and the relation

$$
Y\left(\bar{\psi}, z^{*}\right)=Y\left(e^{z L_{1}} z^{-2 L_{0}} \psi, 1 / z\right)^{\dagger}
$$

holds with $Y$ representing either $V$ or $U$. Note that the operators $W$ satisfy the "intertwining relation"

$$
W(\chi, z) V(\psi, w)=U(\psi, w) W(\chi, z),
$$

with a similar relation holding for $\bar{W}$ (as a consequence of (8) and (9). [Note that this is simply a relation of the form (2) again. This will be a general feature of the following. Any relation of the form (2) or (6) which makes sense (i.e. in which the states are matched to appropriate vertex operators) holds.] 
Consider fixed states $\chi$ and $\rho$ in the representation. Note that we no longer assume that $\chi$ or $\rho$ are equal, real and quasi-primary, as we $\operatorname{did}$ in 12$]$. Then, for $\psi_{j} \in \mathcal{H}$ for $1 \leq j \leq n$, the matrix element

$$
\begin{aligned}
\langle\chi| U\left(\psi_{1}, z_{1}\right) & \ldots U\left(\psi_{n}, z_{n}\right)|\rho\rangle \\
& =\left\langle\chi\left|U\left(\psi_{1}, z_{1}\right) \ldots U\left(\psi_{n-1}, z_{n-1}\right) e^{z_{n} L_{-1}} W\left(\rho,-z_{n}\right)\right| \psi_{n}\right\rangle \text { by }(7) \\
& =\left\langle\chi\left|e^{z_{n} L_{-1}} U\left(\psi_{1}, z_{1}-z_{n}\right) \ldots U\left(\psi_{n-1}, z_{n-1}-z_{n}\right) W\left(\rho,-z_{n}\right)\right| \psi_{n}\right\rangle \text { by (5) } \\
& =\left\langle\chi\left|e^{z_{n} L_{-1}} W\left(\rho,-z_{n}\right) V\left(\psi_{1}, z_{1}-z_{n}\right) \ldots V\left(\psi_{n-1}, z_{n-1}-z_{n}\right)\right| \psi_{n}\right\rangle \text { by (10) } \\
& \equiv\left\langle P\left(\chi, \rho ; z_{n}{ }^{*}\right)\left|V\left(\psi_{1}, z_{1}-z_{n}\right) \ldots V\left(\psi_{n-1}, z_{n-1}-z_{n}\right)\right| \psi_{n}\right\rangle,
\end{aligned}
$$

where

$$
\begin{aligned}
P(\chi, \rho ; z) & =W\left(\rho,-z^{*}\right)^{\dagger} e^{z L_{1}}|\chi\rangle \\
& =\bar{W}\left(e^{-z L_{1}} z^{-2 L_{0}} \bar{\rho},-\frac{1}{z}\right) e^{z L_{1}}|\chi\rangle
\end{aligned}
$$

or, as in [10],

$$
\left\langle P\left(\chi, \rho ; z^{*}\right) \mid \psi\right\rangle=\langle\chi|U(\psi, z)| \rho\rangle .
$$

We can easily modify the equations for $P$ derived in [12] to this more general case. Rather than proceed as in [12], we can simply check the relations directly from the definition (13).

- $\left\langle P\left(\chi, \rho ; z^{*}\right) \mid 0\right\rangle=\langle\chi \mid \rho\rangle$.

- $\left\langle P\left(\chi, \rho ; z^{*}\right)\right|=\left\langle P\left(\chi, \rho ; w^{*}\right)\right| e^{(z-w) L_{-1}}$ : This follows immediately using (5).

- $\langle\overline{P(\rho, \chi ; z)}|=\left\langle P\left(\chi, \rho ; 1 / z^{*}\right)\right| e^{z L_{1}} z^{-2 L_{0}}$ : If we take the inner product with a state $\psi$, the right hand side becomes

$$
\left\langle\chi\left|U\left(e^{z L_{1}} z^{-2 L_{0}} \psi, 1 / z\right)\right| \rho\right\rangle=\left\langle\rho\left|U\left(\bar{\psi}, z^{*}\right)\right| \chi\right\rangle^{*},
$$

using (9). Using the definition again, and the result

$$
\langle\psi \mid \bar{\phi}\rangle=\langle\phi \mid \bar{\psi}\rangle
$$

(see [2]) gives us the left hand side.

- $\overline{P(\chi, \rho ; z)}=P\left(\bar{\chi}, \bar{\rho} ;-z^{*}\right)$ : This is easily verified using the "reality" of the representation [2]

$$
\langle\eta|U(\psi, z)| \phi\rangle^{*}=\langle\bar{\eta}|U(\bar{\psi},-z)| \bar{\phi}\rangle
$$

together with (15).

The claim made in [12], and justified up to technicalities regarding the rigorous definition of the Hilbert space for the representation, is that these equations on $P$ (in the case $\chi=$ $\rho=\bar{\chi}$ ) are also sufficient for it to define a representation (the calculations of [12 show that using (11) as a definition of the vertex operators $U$ satisfies the appropriate properties for a representation, provided the Hilbert space can be understood). The obvious relation to work of Zhu [16] remains to be understood precisely.

In [10], the general solution to the above equations was found, and further it was shown that a necessary (and sufficient) condition for the state $\chi$ to be a ground state in the abstract representation defined via (11) is that $P \equiv P(1)$ be orthogonal to what Zhu denotes by $O(\mathcal{H})$ - a space of states whose zero modes automatically annihilate the ground states of all representations of the $\mathrm{CFT} \mathcal{H}$. 


\section{Construction of intertwiners between non-trivial rep- resentations}

Suppose we have two representations $\mathcal{K}_{1}$ and $\mathcal{K}_{2}$ of a CFT $\mathcal{H}$. We wish to construct the intertwiner representing the action of $\mathcal{K}_{1}$ on $\mathcal{K}_{2}$, i.e. a set of vertex operators corresponding to states in $\mathcal{K}_{1}$ acting on $\mathcal{K}_{2}$. Let us assume that the two representations fuse to give only one representation. The canonical example which we wish to keep in mind is that in which we are considering orbifolding $\mathcal{H}$ with respect to some finite group $G$ (which we take to be abelian for simplicity), and $\mathcal{K}_{1}$ and $\mathcal{K}_{2}$ are $g_{1}$ and $g_{2}$-twisted representations respectively for certain group elements $g_{1}$ and $g_{2}$. (See [4] for definitions and existence theorems on such twisted sectors.) In this case, the intertwiner maps us to a $g_{1} g_{2}$-twisted representation.

The case discussed in the previous section is that in which the intertwiner maps back to $\mathcal{H}$ (the intertwining vertex operators are then simply the $\bar{W}$ 's), i.e. in the case of the orbifold theory where $g_{1}=g_{2}{ }^{-1}$. We consider here the more general case in which the image space is a non-adjoint representation of $\mathcal{H}$. Let us denote it $\mathcal{K}_{3}$.

Choose fixed quasi-primary states $\chi_{1}$ and $\chi_{2}$ in $\mathcal{K}_{1}$ and $\mathcal{K}_{2}$ respectively (typically we will take these to lie in the ground state of the representation - note that we cannot choose them to be real, however, due to the comment following equation (8)), and of respective conformal weights $h_{1}$ and $h_{2}$. Suppose the CFT $\mathcal{H}$ is represented on $\mathcal{K}_{1}, \mathcal{K}_{2}$ and $\mathcal{K}_{3}$ by vertex operators $U_{1}, U_{2}$ and $U_{3}$ respectively. We wish to define an intertwining operator $\hat{W}(\rho, z): \mathcal{K}_{2} \rightarrow \mathcal{K}_{3}$ for $\rho \in \mathcal{K}_{1}$. Using the fact that the representations are irreducible representations of $\mathcal{H}$, all we need to define is the state

$$
\hat{W}\left(U_{1}\left(\psi_{1}, w_{1}\right) \chi_{1}, z\right) U_{2}\left(\psi_{2}, w_{2}\right) \chi_{2}
$$

for all $\psi_{1}, \psi_{2} \in \mathcal{H}$. As argued in [11], this must be given (if we are to have a consistent CFT, i.e. if appropriate intertwining relations are to hold) by

$$
U_{3}\left(\psi_{2}, w_{2}\right) U_{3}\left(\psi_{1}, w_{1}+z\right) \hat{W}\left(\chi_{1}, z\right) \chi_{2} .
$$

Hence, we can identify the required intertwiner once we know $R\left(\chi_{1}, \chi_{2} ; z\right) \equiv \hat{W}\left(\chi_{1}, z\right) \chi_{2}$. In the appendix, we demonstrate that an obvious ansatz at this point cannot work in general. Let us proceed instead as follows.

Expand $R\left(\chi_{1}, \chi_{2} ; z\right)$ as

$$
R\left(\chi_{1}, \chi_{2} ; z\right)=\sum_{n \geq 0} \eta_{n} z^{n-\delta}
$$

$\eta_{n} \in \mathcal{K}_{3}$ (and suppressing the dependence on $\chi_{1}$ and $\chi_{2}$ for the sake of notational compactness), for some shift $\delta$ with $\eta_{0} \neq 0$. The conformal weight of $\eta_{n}$ is $n+h_{3}$, for some constant $h_{3}$. Denote $\eta_{0}$ by $\chi_{3}$. (Note that $\delta=h_{1}+h_{2}-h_{3}$.) [Typically, $\chi_{3}$ will lie in the ground state of $\mathcal{K}_{3}$, though we do not know in general when this must be so and so cannot assume it. This is one of the crucial problems that we have to overcome in the following.]

In order to map $R\left(\chi_{1}, \chi_{2} ; z\right)$ to a state inside the original CFT $\mathcal{H}$, we act with the conjugate intertwining operator $\bar{W}_{3}$ (c.f. (8)), where $W_{3}$ is the intertwiner corresponding to $U_{3}$ as in (1). Following (12), we consider the state

$$
Q\left(\chi_{1}, \chi_{2} ; w, z\right) \equiv \bar{W}_{3}\left(e^{-w L_{1}} w^{-2 L_{0}} \overline{\chi_{3}},-\frac{1}{w}\right) e^{w L_{1}} R\left(\chi_{1}, \chi_{2} ; z\right)
$$


in $\mathcal{H}$. (Note that the state $\chi_{3}$ is, at this point, still unknown.) Another way to look at this is to consider the following matrix elements which, by irreducibility of $\mathcal{K}_{3}$ as a $\mathcal{H}$ representation, are clearly sufficient, if known for all $\psi \in \mathcal{H}$, to determine $R\left(\chi_{1}, \chi_{2} ; z\right)$ completely.

$$
\begin{aligned}
& \left\langle\chi_{3}\left|U_{3}(\psi, w) \hat{W}\left(\chi_{1}, z\right)\right| \chi_{2}\right\rangle \\
= & \left\langle\chi_{2}\left|\hat{W}^{\dagger}\left(\chi_{1}, z\right) U_{3}\left(e^{w^{*} L_{1}} w^{*-2 L_{0}} \bar{\psi}, \frac{1}{w^{*}}\right)\right| \chi_{3}\right\rangle^{*} \quad \text { by (9) } \\
= & \left\langle\chi_{2}\left|\hat{W}^{\dagger}\left(\chi_{1}, z\right) e^{\frac{1}{w^{*}} L_{-1}} W_{3}\left(\chi_{3},-\frac{1}{w^{*}}\right) e^{w^{*} L_{1}} w^{*-2 L_{0}}\right| \bar{\psi}\right\rangle^{*} \quad \text { c.f. (17) } \\
= & \left\langle\bar{\psi}\left|w^{-2 L_{0}} e^{w L_{-1}} \bar{W}_{3}\left(e^{-\frac{1}{w} L_{1}} w^{2 L_{0}} \overline{\chi_{3}},-w\right) e^{\frac{1}{w} L_{1}} W\left(\chi_{1}, z\right)\right| \chi_{2}\right\rangle \quad \text { by (8) } \\
= & \left\langle\bar{\psi}\left|w^{-2 L_{0}} e^{w L_{-1}}\right| Q\left(\chi_{1}, \chi_{2} ; 1 / w, z\right)\right\rangle .
\end{aligned}
$$

[The expression (20) may be simplified slightly by noting that $\chi_{3}$ is necessarily quasi-primary (in fact, it must be primary), i.e.

$$
\begin{aligned}
Q\left(\chi_{1}, \chi_{2} ; w, z\right) & \equiv \bar{W}_{3}\left(w^{-2 L_{0}} \overline{\chi_{3}},-\frac{1}{w}\right) e^{w L_{1}} \hat{W}\left(\chi_{1}, z\right) \chi_{2} \\
& =w^{-2 h_{3}}\left(\frac{z}{1-w z}\right)^{2 h_{1}} \overline{W_{3}}\left(\overline{\chi_{3}},-\frac{1}{w}\right) \hat{W}\left(\chi_{1}, \frac{z}{1-w z}\right) \chi_{2}
\end{aligned}
$$

using the $s u(1,1)$ transformation properties of primary fields from e.g. [7].]

Hence, knowledge of the state $Q\left(\chi_{1}, \chi_{2} ; w, z\right) \in \mathcal{H}$ is sufficient to determine the intertwining operators $\hat{W}(\rho, z)$. But we can say much more. Rather than just treating $Q$ as another state in $\mathcal{H}$ to be determined by equations analogous to those we have to solve for the $P$ 's, we shall show that $Q$ is determined uniquely in terms of the $P$ 's corresponding to the representations $\mathcal{K}_{1}$ and $\mathcal{K}_{2}$.

Inserting the expansion (19) of $R\left(\chi_{1}, \chi_{2} ; z\right)$ in (20), we have

$$
Q\left(\chi_{1}, \chi_{2} ; w, z\right)=\sum_{n \geq 0} z^{n-\delta} P\left(\eta_{n}, \chi_{3} ; w\right) .
$$

Let us first use this to simplify (21). We obtain, using the equations satisfied by the $P$ 's described in the previous section,

$$
\begin{aligned}
\left\langle\chi_{3}\left|U_{3}(\psi, w) \hat{W}\left(\chi_{1}, z\right)\right| \chi_{2}\right\rangle & =\sum_{n \geq 0} z^{n-\delta}\left\langle\bar{\psi}\left|w^{-2 L_{0}} e^{w L_{-1}}\right| P\left(\eta_{n}, \chi_{3} ; 1 / w\right)\right\rangle \\
& =\sum_{n \geq 0} z^{n-\delta}\left\langle\bar{\psi} \mid \overline{P\left(\chi_{3}, \eta_{n} ; w^{*}\right)}\right\rangle \\
& =\sum_{n \geq 0} z^{n-\delta}\left\langle P\left(\chi_{3}, \eta_{n} ; w^{*}\right) \mid \psi\right\rangle
\end{aligned}
$$

by (15) (in fact, obvious from the definition of the $P$ 's).

Consider the matrix elements

$$
\left\langle R\left(\chi_{1}, \chi_{2} ; d^{*}\right)\left|U_{3}(\psi, w)\right| R\left(\chi_{1}, \chi_{2} ; z\right)\right\rangle
$$

for arbitrary states $\psi \in \mathcal{H}$. By the series expansion of $R$ and the definition of $P$, this is simply

$$
\sum_{m, n \geq 0} d^{m-\delta} z^{n-\delta}\left\langle\eta_{m}\left|U_{3}(\psi, w)\right| \eta_{n}\right\rangle=\sum_{m, n \geq 0} d^{m-\delta} z^{n-\delta}\left\langle P\left(\eta_{m}, \eta_{n} ; w^{*}\right) \mid \psi\right\rangle
$$


On the other hand, (25) is

$$
\begin{aligned}
& \left\langle\chi_{2}\left|\hat{W}\left(\chi_{1}, d^{*}\right)^{\dagger} U_{3}(\psi, w) \hat{W}\left(\chi_{1}, z\right)\right| \chi_{2}\right\rangle \\
= & d^{-2 h_{1}}\left\langle\chi_{2}\left|\overline{\hat{W}}\left(\bar{\chi}_{1}, \frac{1}{d}\right) \hat{W}\left(\chi_{1}, z\right) U_{2}(\psi, w)\right| \chi_{2}\right\rangle,
\end{aligned}
$$

by an appropriate intertwining relation and the analog of (8),

$$
=d^{-2 h_{1}}\left\langle\chi_{2}\left|U_{2}\left(\overline{W_{1}}\left(\overline{\chi_{1}}, \frac{1}{d}-z\right) \chi_{1}, z\right) U_{2}(\psi, w)\right| \chi_{2}\right\rangle,
$$

by an appropriate analog of (6),

$$
=(1-d z)^{-2 h_{1}}\left\langle\chi_{2}\left|U_{2}(\psi, w) U_{2}\left(P\left(\chi_{1}, \chi_{1} ; \frac{d}{d z-1}\right), z\right)\right| \chi_{2}\right\rangle,
$$

by the definition of $P$,

$$
=(1-d z)^{-2 h_{1}}\left\langle P\left(\chi_{2}, \chi_{2} ; z^{*}\right)|V(\psi, w-z)| P\left(\chi_{1}, \chi_{1} ; d /(d z-1)\right)\right\rangle,
$$

by (11). Thus we may evaluate (26) in terms of the known $P$ 's corresponding to the representations $\mathcal{K}_{1}$ and $\mathcal{K}_{2}$, i.e. we can, in principle, calculate $P\left(\eta_{m}, \eta_{n} ; z\right) . Q\left(\chi_{1}, \chi_{2} ; w, z\right)$ itself is simply a series in $P\left(\eta_{n}, \eta_{0} ; w\right)$, and thus is fixed. Alternatively, we can regard the matrix element on the left hand side of (21) as being given by the $d^{-\delta}$ term in the expansion of (30) about $d=0$ (obvious from the definitions without resorting to the expansions in terms of states $\eta_{n}$, but the approach in terms of the $\eta_{n}$ 's bears a closer correspondence to the earlier work), or rather of

$$
z^{-h_{\psi}} z^{-\delta}\left\langle P\left(\chi_{2}, \chi_{2} ; 1\right)\left|V\left(\psi, \frac{w}{z}-1\right)\right| P\left(\chi_{1}, \chi_{1} ; d\right)\right\rangle,
$$

where $h_{\psi}$ is the conformal weight of $\psi$. We conclude therefore that the intertwiner representing the action of $\mathcal{K}_{1}$ on $\mathcal{K}_{2}$ is determined. The only ambiguity is in identifying the state $\chi_{3}$ in the representation $\mathcal{K}_{3}$. If no explicit structure for $\mathcal{K}_{3}$ is known, it may simply be built up from the state $\chi_{3}$, using our knowledge of $P\left(\chi_{3}, \chi_{3} ; z\right)$ and (11) via the arguments of [12]. On the other hand, if we already have an explicit realization of $\mathcal{K}_{3}$, we can typically identify $\chi_{3}$ by the way in which the Hilbert space defined by (11) is built up from it (e.g. at the simplest level, we can easily calculate its conformal weight). In typical applications, $\chi_{3}$ will simply lie in the ground state of the target representation. Also note that, in cases where we have an explicit presentation of $\mathcal{K}_{3}$, it is often easier to use the above as a means of defining $R\left(\chi_{1}, \chi_{2} ; z\right)$ rather than $Q\left(\chi_{1}, \chi_{2} ; w, z\right)$, which is, in some sense, a more fundamental state and can be read off from comparing the left hand side of (24) to the leading term in the expansion of (31).

As a final comment in this section, it is interesting to note, from comparing the definition of $P$ with the form of (30), that the intertwiner between (non-trivial) modules is described entirely by the state

$$
P\left(P\left(\chi_{2}, \chi_{2} ; 1\right), P\left(\chi_{1}, \chi_{1}, w\right) ; z\right)
$$




\section{Examples and Application to Orbifolds}

As an illustration of the above points, let us first consider the trivial example of representations of the Heisenberg algebra. As usual, we take this to be built up from a vacuum $|0\rangle$ by the action of creation and annihilation operators satisfying

$$
\begin{aligned}
{\left[a_{m}, a_{n}\right] } & =m \delta_{m,-n} \quad \text { for } m, n \in \mathbf{Z} \\
a_{n}|0\rangle & =0 \text { for } n \geq 0
\end{aligned}
$$

[As is well known, appropriate vertex operators and an appropriate conjugation can be defined to make this a bosonic, hermitian, meromorphic conformal field theory of unit central charge.] Representations, labelled $\mathcal{K}_{\lambda}$, are built up by an isomorphic set of operators (we will identify them) acting on a "momentum state" $|\lambda\rangle$ of conformal weight $\frac{1}{2} \lambda^{2}, \lambda \in \mathbf{R}$, satisfying

$$
\begin{array}{r}
a_{n}|\lambda\rangle=0 \quad \text { for } n>0 \\
a_{0}|\lambda\rangle=\lambda|\lambda\rangle .
\end{array}
$$

[Again, appropriate vertex operators may easily be defined.] We can show that

$$
P(|\lambda\rangle,|\mu\rangle ; z)=\delta_{\lambda \mu} e^{-\lambda \sum_{n>0}(-1)^{n} \frac{a_{-n}}{n} z^{-n}}|0\rangle,
$$

either from the explicit form of the vertex operators defining the representation or, more appropriately for the point of view taken in this paper, from solving the appropriate equations for $P$ as in [10].

Suppose that we wished to consider the intertwiner representing the action of $\mathcal{K}_{\lambda}$ on $\mathcal{K}_{\mu}$. From the known structure of the representations, the fusion results in the representation $\mathcal{K}_{\lambda+\mu}$ and the state $\chi_{3}$ in this instance is simply proportional to the ground state $|\lambda+\mu\rangle$ (we denote the constant of proportionality $\epsilon(\lambda, \mu)$ - see e.g. [3] for an explanation of such factors). Consider a typical matrix element involving the intertwiner. Suppose we wish to evaluate

$$
\langle\lambda+\mu| \epsilon(\lambda, \mu)^{*} U\left(a_{-1}|0\rangle, w\right) W(|\lambda\rangle, z)|\mu\rangle,
$$

where we have dropped suffices from vertex operators for the sake of clarity. Using the known structure of the vertex operators, this turns out to be

$$
z^{\lambda \mu}\left(\frac{\lambda}{w-z}+\frac{\mu}{w}\right)
$$

Alternatively, by the above, we may calculate this without any direct knowledge of the structure of the representations or their vertex operators, merely of the underlying Heisenberg algebra. We simply evaluate

$$
\left\langle P\left(|\mu\rangle,|\mu\rangle ; z^{*}\right)\right| V\left(a_{-1}|0\rangle, w-z\right)|P(|\lambda\rangle,|\lambda\rangle ; d /(d z-1))\rangle .
$$

This gives

$$
(d z)^{\lambda \mu}\left(\frac{\lambda}{w-z}\left(\frac{1-d z}{1-d w}\right)+\frac{\mu}{w}\right) .
$$

Looking at the leading term in the expansion in $d$ gives (37) (and also shows that the conformal weight of the state " $\chi_{3}$ " is $\frac{1}{2}(\lambda+\mu)^{2}$, from the leading power of $d(i . e . \delta=-\lambda \mu)$. 
Note that an alternative trivial means of directly calculating the conformal weight of the state $\chi_{3}$ in general (and also the norms of the states $\eta_{n}$ in the expansion of $R$ ) is to simply use the main result of the preceding section with $\psi=|0\rangle$, i.e. the identity

$$
\sum_{n \geq 0} \alpha^{n-\delta}\left\|\eta_{n}\right\|^{2}=(1-\alpha)^{-2 h_{1}}\left\langle P\left(\chi_{2}, \chi_{2} ; 1\right) \mid P\left(\chi_{1}, \chi_{1} ; \alpha /(\alpha-1)\right)\right\rangle,
$$

(setting $\alpha=d z$ and using the fact that $P(z)=z^{-L_{0}} P(1)$ ).

Before proceeding to the third order orbifold example, some general comments are in order. Firstly, we note trivially that the last two of the four equations for $P$ derived in section 2 give

$$
\left\langle P\left(\bar{\chi}, \bar{\rho} ;-z^{*}\right)\right|=\left\langle P\left(\chi, \rho, \frac{1}{z^{*}}\right)\right| e^{z L_{1}} z^{-2 L_{0}} .
$$

Suppose that we are orbifolding a CFT $\mathcal{H}$ with respect to some discrete group $G$ with $N$ non-trivial conjugacy classes. We obtain one twisted sector for each of these classes [8]. Let us choose states $\chi_{i}, 1 \leq i \leq N$, in the ground states of the sector twisted by a group element $g_{i}$. Further, choose these states so that $\chi_{i}=\overline{\chi_{j(i)}}$ where $g_{j(i)}{ }^{-1}$ lies in the conjugacy class of $g_{i}$. Set $P_{i}(z)=P\left(\chi_{i}, \chi_{i}, z\right)$. Then, $P_{j(i)}(z)$ is determined in terms of $P_{i}(z)$ by (41).

We will argue below, at least in the case $G=\mathbf{Z}_{3}$, that once the $P_{i}$ 's are specified we need only consider the matrix elements

$$
\left\langle P_{i}(1)|V(\psi, w)| P_{j}(z)\right\rangle,
$$

for all $\psi \in \mathcal{H}$ and all $1 \leq i \leq j \leq N$ in order to specify the orbifold theory (and check its consistency) completely. By the above observation, we may restrict $i$ and $j$ to a certain subset of $\{1,2, \ldots, N\}$ (i.e. a minimal subset $S$ such that $S \cup j(S)=\{1,2, \ldots, N\}$ ).

Consider then the case of orbifolding with respect to a no-fixed-point third order lattice automorphism the conformal field theory corresponding to an even self-dual lattice $\Lambda$. In this case, there are two non-trivial conjugacy classes (twisted sectors).

In order to be more specific, let us give an explicit realisation of the theory. [See [1] for full details.] For notational convenience, we rewrite the even self-dual lattice admitting a third order no-fixed-point automorphism in terms of a complex lattice $\Lambda$ over the ring of Eisenstein integers and of (complex) dimension $d$ (a multiple of 4). The CFT (before orbifolding) has Hilbert space $\mathcal{H}(\Lambda)$ built up from "momentum" states $|\lambda\rangle, \lambda \in \Lambda$, by the action of creation and annihilation operators $b_{n}^{i}$ and $\bar{b}_{n}^{i}, n \in \mathbf{Z}-\{0\}, 1 \leq i \leq d$, satisfying

$$
\begin{array}{r}
{\left[b_{m}^{i}, \bar{b}_{n}^{j}\right]=m \delta_{m,-n} \delta^{i j} ; \quad\left[b_{m}^{i}, b_{n}^{j}\right]=\left[\bar{b}_{m}^{i}, \bar{b}_{n}^{j}\right]=0} \\
b_{m}^{i}|\lambda\rangle=\bar{b}_{n}^{j}|\lambda\rangle=0 \text { for } m, n>0 .
\end{array}
$$

Appropriate vertex operators and conjugation may be defined to make this a hermitian, meromorphic, bosonic conformal field theory of central charge $2 d$ (which we also denote $\mathcal{H}(\Lambda)$ ). It admits an obvious third order automorphism $\theta$ induced by the third order automorphism $\lambda \mapsto \omega \lambda, \omega=e^{2 \pi i / 3}$, on the lattice. We orbifold with respect to the action of this $\mathbf{Z}_{3}$.

The two twisted sectors have identical Hilbert spaces. In order to construct them, we introduce a set of "twisted" oscillators $c_{r}^{i}, 1 \leq i \leq d, r \in \mathbf{Z} \pm \frac{1}{3}$, satisfying

$$
\left[c_{r}^{i}, c_{s}^{j}\right]=r \delta_{r,-s} \delta^{i j} .
$$


Then we construct the Hilbert space $\mathcal{H}_{1}$ built up by the action of these on a ground state space $\mathcal{G}$ annihilated by $c_{r}^{i}$ for $r>0$. $\quad[\mathcal{G}$ is essentially a representation space for a set of cocycle operators required for consistency of the theory - see [11] for more details.] Define a second space $\mathcal{H}_{2}$ built up using an isomorphic set of oscillators $\bar{c}_{r}^{i}$. [Note that, though one of the key points of this paper is that such explicit constructions are, in general, unnecessary, we wish to relate to earlier work and some details of the twisted sectors of the orbifold theory are required.]

$\mathcal{H}_{1}$ and $\mathcal{H}_{2}$ can be given the structure of non-meromorphic modules for the CFT $\mathcal{H}(\Lambda)$. [In defining the action of the CFT on the modules, there is essentially a choice in associating states composed of $b$ oscillators with states built up from twisted oscillators graded by $\mathbf{Z}+\frac{1}{3}$ (and those composed of $\bar{b}$ oscillators with states built up from twisted oscillators graded by $\mathbf{Z}-\frac{1}{3}$ ) or with states built up from twisted oscillators graded by $\mathbf{Z}-\frac{1}{3}$ (and conversely for $\bar{b}$ ) - we make opposite choices for $\mathcal{H}_{1}$ and $\mathcal{H}_{2}$.] The ground state $\mathcal{G}$ turns out (in both modules) to have conformal weight $d / 9$. The oscillators $c_{r}^{i}$ (or $\bar{c}_{r}^{i}$ ) reduce the conformal weight by $r$ as usual. Let $\mathcal{H}_{1}^{0}, \mathcal{H}_{2}^{0}$ be the projections of the modules on to states of integral conformal weight (we must take $d$ to be a multiple of 12 ). It is easily shown that these form irreducible meromorphic modules for the sub-CFT $\mathcal{H}(\Lambda)^{0}$ given by the $\theta=1$ sector of $\mathcal{H}(\Lambda)$.

The hypothesis is then that $\widetilde{\mathcal{H}}(\Lambda) \equiv \mathcal{H}(\Lambda)^{0} \oplus \mathcal{H}_{1}^{o} \oplus \mathcal{H}_{2}^{0}$ can be given the structure of a hermitian, meromorphic, bosonic conformal field theory. What we have shown in the preceding section is that there is at most one way in which this may be achieved, i.e. the intertwiner between the two twisted sectors required to complete the structure to a CFT can be defined in only one way in terms of the $P^{\prime}$ 's corresponding to the modules $\mathcal{H}_{1}^{0}$ and $\mathcal{H}_{2}^{0}$. Further, we have shown how the matrix elements of the intertwiner may be evaluated.

In [11], we conjectured an ansatz for the form of the states in $\mathcal{H}_{2}^{0}$ given by the action of the intertwiner corresponding to a state $\chi$ in the ground state of $\mathcal{H}_{1}^{0}$ on itself, i.e. the state $R(\chi, \chi ; z)$ in the notation of the previous section, for $d$ a multiple of 36 (so that $\mathcal{G} \subset \mathcal{H}_{1,2}^{0}$ ). This is seen to be no longer necessary, and may be checked by verifying the equality of (30) with $\chi_{1}=\chi_{2}=\chi$ and $\left\langle R\left(\chi, \chi ; d^{*}\right)\left|U_{2}(\psi, w)\right| R(\chi, \chi ; z\rangle\right.$, where the vertex operators $U_{2}$ give the representation of $\mathcal{H}(\Lambda)^{0}$ on $\mathcal{H}_{2}^{0}$, for all $\psi \in \mathcal{H}(\Lambda)^{0}$. [Note that due to a slight change of notation here, the state " $\chi_{3}$ " lies in $\mathcal{H}_{2}$, and so the vertex operators labelled $U_{3}, W_{3}$ previously are here denoted $U_{2}, W_{2}$.]

In particular, it was assumed that the ground state of $\mathcal{H}_{2}^{0}$ occurred in the expansion of $R(\chi, \chi ; z)$, i.e. that the coefficient $\delta$ of the previous section is given by the conformal weight, $d / 9$, of the twisted sector ground states. This may be easily checked, as remarked above, by simply evaluating $\langle P(\chi, \chi ; 1) \mid P(\chi, \chi ; d)\rangle$ and expanding around $d=0$.

It was observed in [11] that a total of 18 locality relations need to be checked in order to verify that $\widetilde{\mathcal{H}}(\Lambda)$ is consistent as a CFT. Most of these follow from locality in $\mathcal{H}(\Lambda)$, the fact that $\mathcal{H}_{1,2}^{0}$ form meromorphic irreducible representations of $\mathcal{H}(\Lambda)^{0}$ and suitable definitions of conjugation in the twisted sectors. There remain to be proved four relations, which we now translate into our current notation.

1. $W_{1}(\chi, z) \overline{W_{2}}(\chi, w)=W_{1}(\chi, w) \overline{W_{2}}(\chi, z)$.

Take a matrix element between states $\langle\chi|$ and $U_{2}(\psi, \rho)|\bar{\chi}\rangle$ for arbitrary $\psi \in \mathcal{H}(\Lambda)^{0}$ (note that $\bar{\chi} \in \mathcal{H}_{2}^{0}$ ). Note that no information is lost by doing this because of the irreducibility of the representations and the fact that the intertwining and duality relations we already have allow us to use the vertex operator corresponding to the 
state $\psi$ to raise the ground states to an arbitrary state in the corresponding sectors. Moving the $U_{2}$ into the middle as a $V$ by use of an intertwining relation and using the definition of $P$ gives us the relation

$$
w^{-2 \delta}\left\langle P\left(\chi, \chi ;-z^{*}\right)|V(\psi, \rho)| P\left(\bar{\chi}, \bar{\chi} ;-\frac{1}{w}\right)\right\rangle=z^{-2 \delta}\left\langle P\left(\chi, \chi ;-w^{*}\right)|V(\psi, \rho)| P\left(\bar{\chi}, \bar{\chi} ;-\frac{1}{z}\right)\right\rangle,
$$

or, more compactly,

$$
G(\chi, \psi ; \alpha, \beta)=\beta^{h_{\psi}-2 \delta} G\left(\chi, \psi ; \alpha \beta, \frac{1}{\beta}\right)
$$

for all $\psi \in \mathcal{H}(\Lambda)^{0}$, where $h_{\psi}$ is the conformal weight of $\psi$ and

$$
G(\chi, \psi ; \alpha, \beta) \equiv\langle P(\chi, \chi ; 1)|V(\psi, \alpha)| P(\bar{\chi}, \bar{\chi} ; \beta)\rangle
$$

[We use $x^{L_{0}} V(\psi, \rho) x^{-L_{0}}=x^{h_{\psi}} V(\psi, z \rho)$ and $x^{-L_{0}} P(\chi, \chi ; z)=P(\chi, \chi ; x z)$.]

2. $\hat{W}(\chi, z) \chi=e^{z L_{-1}} \hat{W}(\chi,-z) \chi$.

This "skew-symmetry" relation (an analog of (7)) on the state $R(\chi, \chi ; z)$ could be translated into a corresponding requirement on the state $Q \in \mathcal{H}(\Lambda)^{0}$. However, the philosophy which we are trying to pursue here is to check everything in terms of matrix elements of the form $(30)$. So we choose to express matrix elements of $R$ as the $d^{-\delta}$ term in the expansion of (30) around $d=0$, and hence find that our locality relation is equivalent to the requirement that

$$
O(F(\chi, \psi ; \alpha, \beta), \beta, \delta)=(-1)^{h_{\psi}+\delta} O(F(\chi, \psi ;-\alpha-1, \beta), \beta, \delta),
$$

where $O(H(\beta), \beta, \delta)$ denotes the $\beta^{-\delta}$ term in the expansion of $H(\beta)$ around $\beta=0$ and

$$
F(\chi, \psi ; \alpha, \beta) \equiv\langle P(\chi, \chi ; 1|V(\psi, \alpha)| P(\chi, \chi ; \beta)\rangle .
$$

3. $\overline{W_{2}}(\chi, z) \hat{W}(\chi, w)=\overline{W_{2}}(\chi, w) \hat{W}(\chi, z)$.

(Recall, as remarked above, in the notation of section 3, rather unfortunately, $\overline{W_{2}}$ is $\overline{W_{3}}$ !) Acting on the state $\chi$ with this relation gives us a symmetry of the state $Q(\chi, \chi ; w, z)$, provided we have $\overline{\chi_{3}}=\chi$. There are two steps to this. First, we must use the above result that $\chi_{3}$, the leading term in the expansion of $R(\chi, \chi ; z)$ about $z=0$, lies in the ground state of $\mathcal{H}_{2}^{0}$, i.e. check one of the assumptions of the ansatz of [11] by confirming $\delta=d / 9$. Secondly, we then simply choose a "gauge" in which the spinor states are such that $\overline{\chi_{3}}=\chi$ by suitable redefinition of $\chi$ by action of an appropriate unitary matrix.

The locality requirement then becomes, from (22), the requirement of symmetry of $Q\left(\chi, \chi ;-\frac{1}{z}, \frac{z w}{z-w}\right)$ under $w \leftrightarrow z$.

As we have remarked above, however, we wish to translate everything into requirements on the matrix elements given by the functions $F$ and $G$ defined above. We therefore use the identity (21) and the relation between (24) and (30) to rewrite the locality 
relation as a requirement for symmetry under $w \leftrightarrow z$ of the $d^{-\delta}$ term in the expansion about $d=0$ of

$$
\left\langle P\left(\chi, \chi ;\left(\frac{z w}{z-w}\right)^{*}\right)\left|V\left(z^{2 L_{0}} e^{-z L_{1}} \bar{\psi}, \frac{z^{2}}{w-z}\right)\right| P(\chi, \chi ; d)\right\rangle,
$$

for all $\psi \in \mathcal{H}(\Lambda)^{0}$. It clearly suffices to take $\psi$ quasi-primary. Then, with some trivial manipulations, we see that we require, for all quasi-primary $\psi \in \mathcal{H}(\Lambda)^{0}$ of conformal weight $h_{\psi}$,

$$
O\left(F\left(\chi, \psi ; \frac{1}{\alpha}, \beta\right), \beta, \delta\right)=(-1)^{h_{\psi}+\delta} \alpha^{2 h_{\psi}} O(F(\chi, \psi ; \alpha, \beta), \beta, \delta) .
$$

4. $\overline{\hat{W}}(\bar{\chi}, z) \hat{W}(\chi, w)=W_{2}(\chi, w) \overline{W_{1}}(\bar{\chi}, z)$.

Taking suitable matrix elements, as above, this is simply (making a simple change of variables and writing the left hand side in terms of $R$ )

$$
\left\langle R\left(\chi, \chi ; d^{*}\right)\left|U_{2}(\psi, w)\right| R(\chi, \chi ; z)\right\rangle=\left\langle P\left(\chi, \chi ;-z^{*}\right)|V(\psi, w)| P(\chi, \chi ;-d)\right\rangle
$$

or

$$
\left\langle R\left(\chi, \chi ; d^{*}\right)\left|U_{2}(\psi, w)\right| R(\chi, \chi ; z)\right\rangle=(-z)^{-h_{\psi}} F\left(\chi, \psi ;-\frac{w}{z}, d z\right)
$$

We have reduced verification of the consistency of $\widetilde{\mathcal{H}}(\Lambda)$ to checking the four relations (46), (48), (51) and (53) in terms of the functions $F$ and $G$. ( $R$ is defined by $F$, as discussed previously, and in principle (53) can be checked - we will comment further on this below however.)

It should be stressed that checking the consistency of the orbifold theory is in any case a non-trivial requirement. For example, in the case of a reflection-twisted orbifold of the theory $\mathcal{H}(\Sigma)$ corresponding to an even lattice $\Sigma[3]$, the orbifold is only consistent for $\sqrt{2} \Sigma^{*}$ an even lattice. A similar condition must hold in this case, though presumably our restriction to consideration of an even self-dual lattice renders the $\mathbf{Z}_{3}$-twisted orbifold theory consistent.

Some parts of the calculation of $F$ and $G$ were performed in [11]. We have, in the case $d=0 \bmod 36($ so that $\chi$ is a pure spinor ground state, i.e. $\chi \in \mathcal{G}$ )

$$
P(\chi, \chi ; z)=\sum_{\lambda \in \Lambda}\left\langle\chi\left|\gamma_{\lambda}\right| \chi\right\rangle 3^{-\frac{3}{2} \lambda^{2}} z^{-\lambda^{2}} e^{\sum_{\substack{n, m \geq 0 \\ n+m>0}} A_{m n} b_{-m} \cdot \bar{b}_{-n} z^{-m-n}}|\lambda\rangle,
$$

(for some suitable "cocycles" $\gamma_{\lambda}$ acting on the spinor ground state in which $\chi$ lies) where

$$
A_{m n}=\frac{1}{m+n}\left(\begin{array}{c}
-\frac{1}{3} \\
m
\end{array}\right)\left(\begin{array}{c}
-\frac{2}{3} \\
n
\end{array}\right) \text {. }
$$

$P(\bar{\chi}, \bar{\chi} ; z)$ is given by the same expression but with what turns out to be the important difference that the matrix $A$ is transposed. In evaluating the function $F$, we are faced with such expressions as $\operatorname{det}\left(1-A A^{T}\right)\left(\right.$ since $\left.b_{n}^{\dagger}=\bar{b}_{-n}\right)$, whereas $G$ involves $\operatorname{det}\left(1-A^{2}\right)$. As remarked in [11], the former is problematic, and its solution could only be conjectured, whereas the latter was calculated explicitly (in appendix $\mathrm{C}$ of [11]) by simply adapting the 
arguments of [3], which in turn follow from earlier calculations performed in the context of dual models [1, 15].

We see now from our observation (41) (due to the "reality" of the representation) that $F$ can be evaluated in terms of $G$ and much of the calculation proposed in [11 is unnecessary.

In fact, we have

$$
F(\chi, \psi ; \alpha, \beta)=(1-\beta)^{-h_{\psi}}(-\beta)^{h_{\psi}} G\left(\chi, \psi ; \frac{\alpha \beta+1}{\beta-1}, 1-\beta\right),
$$

and we simply check the relations (46), (48), (51) and (53) in terms of the single function $G$. In fact, (48) follows easily from (46) as a consequence of (56) (actually a non-limiting form of (48), i.e. with the $O(\cdot)$ removed, holds). This, together with the following important conjecture, is a crucial simplification of the orbifold analysis.

We conjecture that (53) should hold as a consequence of our definition of $R$. First note that the identity between (30) and the left hand side of (53) derived earlier is consistent with (53) if

$$
(1-\beta)^{-2 \delta} F\left(\chi, \psi ;-1-\alpha, \frac{\beta}{\beta-1}\right)=(-1)^{h_{\psi}} F(\chi, \psi ; \alpha, \beta) .
$$

Translating this into a requirement on $G$ via $(56)$ it is no more than (46). Now, the matrix elements of $R$ are defined by the leading term in the expansion around $d=0$ of the right hand side of (30) (or (53)), and hence so is the left hand side of (53). In other words, we should be able to deduce $F(\chi, \psi ; \alpha, \beta)$ from the $\beta^{-\delta}$ term in the expansion about $\beta=0$. This is certainly clear when $\delta=0$, since derivatives of $F$ with respect to $\beta$ are implemented by simple insertion of an appropriate Virasoro mode, i.e. considering $\psi$ replaced by $V\left(\psi_{L}, z\right) \psi$. The case when $\delta \neq 0$ is not so clear, and though intuitively we expect this still to hold, many details remain to be checked. Even then, the implication that (53) follows from this property needs still to be established rigorously, and clearly ties in with the still to be proven definition of a module in terms of the state $P$ sketched in [12]. This is the subject of further work.

[Some more weight may be lent to these conjectures by the following observations. We note that the state $R(\chi, \chi ; z)$ is itself of the form of a "P" state, though of a more generalised form since it lies in a non-adjoint module. It obeys similar equations, (provided the orbifold is consistent) and by considering the matrix element

$$
\left\langle R(\chi, \chi ; 1)\left|e^{-L_{1}} U_{2}(\psi, w)\right| R(\chi, \chi ; z)\right\rangle
$$

for $\psi$ quasi-primary and acting with the exponential to the right or to the left [9] we can simply deduce the identity

$$
\left\langle R(\chi, \chi ;-1)\left|U_{2}(\psi, w)\right| R(\chi, \chi ; z)\right\rangle=(1+w)^{-2 h_{\psi}}(1+z)^{-2 \delta}\left\langle R(\chi, \chi ; 1)\left|U_{2}\left(\psi, \frac{w}{w+1}\right)\right| R\left(\chi, \chi ; \frac{z}{z+1}\right)\right\rangle .
$$

This is a potential "hidden" symmetry, which must lead to a symmetry of $F$ and hence $G$ via (53). If such an identity were non-trivial it would be very mysterious and cast serious doubt on any claims that (53) is a simple consequence of the definition of $R$ - since it would then follow from properties of $R$ in the twisted module that were not apparent at the level of the untwisted sector. Fortunately, however, the symmetry one derives is again the identity (46).] 
Modulo these assumptions then, we see that verification of locality is reduced to simply checking the two relations (46) and (51) inside $\mathcal{H}(\Lambda)^{0}$. We stress once more that, in keeping with our philosophy throughout this paper, no calculations in the twisted sectors are necessary (and even if we were to consider (53) explicitly, the structure of the module in which $R$ lies is induced by the matrix elements given by $F$, as discussed in the last section).

Note also that no assumptions on the form of the intertwiner between the twisted sectors need be made. Indeed, we may check the ansatz given in [11] (for $d$ a multiple of 36)

$$
R(\chi, \chi ; z)=z^{-d / 9} e^{\sum_{r \in \mathbf{Z}+\frac{1}{3}, s \in \mathbf{Z}+\frac{2}{3}} D_{r s} \bar{c}_{-r} \cdot \bar{c}_{-s}(-z)^{r+s}} \chi_{3},
$$

where

$$
D_{r s}=\frac{1}{r+s}\left(\frac{1}{3 s}-1\right)\left(\begin{array}{c}
-\frac{2}{3} \\
r-\frac{1}{3}
\end{array}\right)\left(\begin{array}{c}
-\frac{1}{3} \\
s-\frac{2}{3}
\end{array}\right),
$$

(for some ground state $\chi_{3} \in \mathcal{H}_{2}^{0}$ ), by use of our relation

$$
\begin{aligned}
\left\langle\chi_{3}\left|U_{2}(\psi, w)\right| R(\chi, \chi ; z)\right\rangle & =O\left(z^{-h_{\psi}} z^{-\delta} F\left(\chi, \psi ; \frac{w}{z}-1, d\right) ; d, \delta\right) \\
& =O\left(z^{-h_{\psi}} z^{-\delta}(-d)^{h_{\psi}} G\left(\chi, \psi ; \frac{w d}{z(1-d)}+1,1-d\right) ; d, \delta\right)
\end{aligned}
$$

(where $\delta$ is given by the order of the leading pole in $F$ ).

For $d \equiv 12,24 \bmod 36$, the correct from of $R(\chi, \chi ; z)$ was unclear in 11 and an appropriate ansatz could not be made. Our present reformulation sidesteps this problem by allowing one to calculate $R$ if desired, but moreover arguing that such expressions are unnecessary in any case and the appropriate $P$ (different for $d \equiv 12,24 \bmod 36$ from that given above, though still easily calculated) is all that is required.

The calculations remaining to check consistency of $\widetilde{\mathcal{H}}(\Lambda)$, i. e. evaluating $G(\chi, \psi ; \alpha, \beta)$ for all $\psi \in \mathcal{H}_{2}^{0}$, are just a straightforward generalisation of those performed in [3]. However, they are clearly outside the scope of this short paper and will be presented in a future publication.

Clearly, the ideas here extend beyond the simple $\mathbf{Z}_{3}$ case which we have used as an illustrative example. We may even analyse orbifolds of arbitrary theories where no explicit realisation of the twisted sectors is apparent, perhaps because, for example, the orbifold does not have the same geometric interpretation as is available in the $\mathbf{Z}_{3}$ case.

\section{Conclusions}

Though the main idea of this paper is, in some sense, a rather trivial rewriting of matrix elements involving intertwiners, the same is true of the earlier work in [12, 10] and yet such work led to highly non-trivial results on the uniqueness of certain twisted representations and very significant conjectures regarding "induced" representations of a conformal field theory from a sub-conformal field theory contained within. Similarly in this paper, we have shown that, in the context of orbifold theories endowed with a hermitian structure, the intertwiners between twisted sectors are unique, as one would clearly expect but a conjecture which had up to now remained unproven in general, and moreover we have given a means of actually calculating matrix elements involving these intertwiners in terms of the starting representations. As before, all calculations are carried out entirely within the underlying 
(twist-invariant, in the case of orbifolds) conformal field theory, and no explicit knowledge of the twisted sectors need be used, or indeed known. A proposed technique for verifying consistency of arbitrary (non-geometric) orbifolds has been laid out.

Also, one can view this technique as a means of generating new representations (in the fusion product) from given ones. Our calculations produce the state $P\left(\chi_{3}, \chi_{3} ; z\right)$ from which we may construct the representation $\mathcal{K}_{3}$ as in [12]. This obviously leads us to consider the case in which fusion of the two representations $\mathcal{K}_{1}$ and $\mathcal{K}_{2}$ gives rise to more than one irreducible representation (note that we have implicitly appealed to Dong et al's proof of the uniqueness of the twisted representation [4] in the discussion of this paper, i.e. we assume that in fusing a $g$-twisted representation with an $h$-twisted representation the resulting $g h$-twisted representation is unique). Note that (30) is, by definition, simply $\left\langle P\left(\hat{W}\left(\chi_{1}, d^{*}\right) \chi_{2}, \hat{W}\left(\chi_{1}, w, \chi_{2}\right) ; w\right) \mid \psi\right\rangle$ (we may easily recast this into an explicit form for $P$, i.e. not involving inner products, by use of a relation for the vertex operators $V$ analogous to (耳)), constructed solely from the given representations $\mathcal{K}_{1}$ and $\mathcal{K}_{2}$. If their fusion involves more than one irreducible representation, this state $P$ will split into a sum of $P$ 's corresponding to each of these. It would be expected then that a projection onto states orthogonal to $O(\mathcal{H})$ [16] (or equivalently taking inner products with respect to the $P$ 's corresponding to the irreducible representations, if known - i.e. considering matrix elements $\left.\left\langle P_{i}(y)\left|V\left(P_{j}, w\right)\right| P_{k}(z)\right\rangle\right)$ would enable one to deduce the fusion rules. However, this procedure is complicated by the fact that the states appearing in $\hat{W}\left(\chi_{1}, z\right) \chi_{2}$ do not necessarily include ground states in all of the representations which occur, but rather some representations may appear starting at a higher level. The relation to Frenkel and Zhu's calculations of [5] remains to be made clear. This and related questions form the basis of current research.

\section{A Failure of the naive ansatz for the intertwiner}

We demonstrate in this appendix that an obvious but naive ansatz for the form of the intertwiner representing the action of $\mathcal{K}_{2}$ on $\mathcal{K}_{3}$ fails in general. In the process, we derive some very interesting results related to Zhu's algebra. As yet, we do not yet fully understand the significance of these.

Throughout this appendix, $\psi$ will denote a state in $\mathcal{H}$ of conformal weight $h$. Choose $\chi_{1}$ and $\chi_{2}$ to be ground states in $\mathcal{K}_{1}$ and $\mathcal{K}_{2}$ respectively. Then, using the result

$$
\sum_{n=0}^{h}\left(\begin{array}{l}
h \\
n
\end{array}\right)(-1)^{n}\left(\begin{array}{l}
h+n \\
n+s
\end{array}\right)=0,
$$

for $s=1, \ldots, h$ (which is easily checked by induction), we can easily show from (6) that

$$
\left[\sum_{n=0}^{h}\left(\begin{array}{l}
h \\
n
\end{array}\right)(-1)^{n} V(\psi)_{n+1}, V(\phi, z)\right]=0,
$$

for $\phi$ a highest weight state under the action of $\psi$, i.e. $V(\psi)_{n} \phi=0$ for $n>0$.

In particular, applying this in the orbifold conformal field theory, we have

$$
\sum_{n=0}^{h}\left(\begin{array}{l}
h \\
n
\end{array}\right)(-1)^{n} V(\psi)_{n+1} \hat{W}\left(\chi_{1}, z\right) \chi_{2}=0 .
$$


Now we make the "natural" ansatz

$$
\left.\hat{W}\left(\chi_{1}, z\right) \chi_{2}=U_{3}(H(z), z)\right) \chi_{3}
$$

with $\chi_{3}$ as in section 3 (except we now assume it lies in the ground state of $\mathcal{K}_{3}$ ) and $H(z)$ a state in $\mathcal{H}$ to be determined. Duality calculations together with (65) then imply

$$
\sum_{n=0}^{h}\left(\begin{array}{l}
h \\
n
\end{array}\right)(-1)^{n} \sum_{s=-n}^{h}\left(\begin{array}{c}
h+n \\
s+n
\end{array}\right) V(\psi)_{-s+1} H(1)=0 .
$$

An extension of (63), namely

$$
\sum_{n=0}^{h}\left(\begin{array}{l}
h \\
n
\end{array}\right)(-1)^{n}\left(\begin{array}{l}
h+n \\
n-r
\end{array}\right)=-\left(\begin{array}{l}
h \\
r
\end{array}\right)
$$

gives

$$
\sum_{r=0}^{h}\left(\begin{array}{l}
h \\
r
\end{array}\right) V(\psi)_{r+1} H(1)=0 .
$$

Let us briefly recall Zhu's construction of $O(\mathcal{H})$ [16], a subspace of $\mathcal{H}$ whose zero modes annihilate the ground state of any representation of $\mathcal{H} . O(\mathcal{H})$ is constructed as the span of states $O(\psi, \phi)$ for all pairs $\psi, \phi \in \mathcal{H}$, where

$$
O(\psi, \phi)=\sum_{r=0}^{h}\left(\begin{array}{l}
h \\
r
\end{array}\right) V(\psi)_{-r-1} \phi .
$$

Now, (8) implies that $V(\psi)_{r}^{\dagger}=V\left(e^{L_{1}} \bar{\psi}\right)_{-r}$, and so we see that $H(1)$ is orthogonal to the subspace of $O(\mathcal{H})$ spanned by $O(\psi, \phi)$ for all pairs $\psi, \phi \in \mathcal{H}$ with $\psi$ quasi-primary.

We quote a very useful lemma (we omit the trivial proof):

\section{Lemma}

$O\left(L_{-1} \psi, \phi\right)=-h O(\psi, \phi)+\left(L_{0}+L_{-1}\right) O(\psi, \phi)-O\left(\psi,\left(L_{0}+L_{-1}\right) \phi\right)$.

In addition, we can show, using the commutation relations of $s u(1,1)$ with vertex operators detailed in e.g. [7],

$$
\left(L_{1}+L_{0}\right) H(1)=\left(h_{1}+h_{3}-h_{2}\right) H(1) .
$$

But $\left(L_{1}+L_{0}\right) H(1)=\kappa H(1)$ for some scalar $\kappa$, together with the lemma and the fact that $H(1)$ is orthogonal to $O(\psi, \phi)$ for all $\psi$ quasi-primary implies that $H(1)$ is orthogonal to $O(\mathcal{H})$ by induction.

Now, $\left(L_{-1}+L_{0}\right) \phi \in O(\mathcal{H})$ for all $\phi \in \mathcal{H}$. Hence, we must have

$$
\left(L_{1}+L_{0}\right) H(1)=0
$$

contradicting (71) unless $h_{1}+h_{3}=h_{2}$. Typically this is not the case, and so we deduce that the ansatz for the form of $\hat{W}\left(\chi_{1}, z\right) \chi_{2}$ is inconsistent in general. For example, in a $d$-dimensional lattice theory twisted by a third order automorphism $g$ induced by a no-fixedpoint third order automorphism of the lattice the two twisted sectors both have ground states of conformal weight $\frac{d}{18}$ (take $d$ to be a multiple of 72) [11. It happens that when $\chi_{1}$ and $\chi_{2}$ are taken to be identical states in the ground state of the $g$-twisted sector, then the 
corresponding state $\chi_{3}$ lies in the ground state of the $g^{2}$-twisted sector [11]. In this case, our ansatz for the form of $\hat{W}\left(\chi_{1}, z\right) \chi_{2}$ must fail.

However, there are examples in which the ansatz can, and does, hold. For example, consider representations of the $d$-dimensional Heisenberg algebra. These are described by a $d$-dimensional "momentum" vector $\lambda$ corresponding to the ground state of the representation. The corresponding conformal weight is given by $h=\lambda^{2} / 2$. In fusing the representations, the momenta simply add. Hence our ansatz can only make sense for the action of a representation labelled by $\lambda$ on one labelled by $\mu$ if $\lambda^{2}+\lambda \cdot \mu=0$. In one dimension, this is just the statement that $\lambda=-\mu$ and the target representation is simply the adjoint. In fact in this case $\hat{W}\left(\chi_{\lambda}, z\right) \chi_{\mu}=z^{-\lambda^{2}} P\left(\chi_{\lambda}, \chi_{\mu} ; z\right)$, trivially from the definition (12). $\chi_{3}$ is then simply the vacuum $|0\rangle$, and (1) tells us that

$$
H(z)=e^{-z L_{-1}} z^{-\lambda^{2}} P\left(\chi_{\lambda}, \chi_{\mu} ; z\right)
$$

\section{References}

[1] E. Corrigan, P. Goddard, D. Olive, and R.A. Smith. Evaluation of the scattering amplitude for four dual fermions. Nucl. Phys. B67 (1973) 477.

[2] L. Dolan, P. Goddard, and P. Montague. Conformal field theories, representations and lattice constructions. Accepted for publication in Communications in Mathematical Physics, hep-th/9410029, DAMTP-94-80.

[3] L. Dolan, P. Goddard, and P. Montague. Conformal field theory of twisted vertex operators. Nucl. Phys. B338 (1990) 529.

[4] C. Dong, H. Li, and G. Mason. Twisted representations of vertex operator algebras. Santa Cruz preprint, 1995.

[5] I. Frenkel and Y. Zhu. Vertex operator algebras associated to representations of affine and Virasoro algebras. Duke Math. J. 66 (1992) 123-168.

[6] B. Gato. Construction of vertex operators using operator formalism techniques. Nucl. Phys. B322 (1989) 555.

[7] P. Goddard. Meromorphic conformal field theory. In Infinite dimensional Lie algebras and Lie groups: Proceedings of the CIRM-Luminy Conference, page 556, Singapore, 1989. World Scientific.

[8] T.J. Hollowood. Twisted Strings, Vertex Operators and Algebras. PhD thesis, Durham University, 1988.

[9] P.S. Montague. Codes, Lattices and Conformal Field Theories. PhD thesis, Cambridge University, 1991.

[10] P.S. Montague. On the uniqueness of the twisted representation in the $\mathbf{Z}_{2}$ orbifold construction of a conformal field theory from a lattice. Nucl. Phys. B455 (1995) 461490. hep-th/9507085. 
[11] P.S. Montague. Third and higher order NFPA twisted constructions of conformal field theories from lattices. Nucl. Phys. B441 (1995) 337-382. hep-th/9502138.

[12] P.S. Montague. On representations of conformal field theories and the construction of orbifolds. Lett. Math. Phys. 38 (1996) 1-11. hep-th/9507083.

[13] A.N. Schellekens. Classification of ten-dimensional heterotic strings. Phys. Lett. B277 (1992) 277.

[14] A.N. Schellekens. Meromorphic $c=24$ conformal field theories. Comm. Math. Phys. 153 (1993) 159.

[15] J. Schwarz and C.C. Wu. Off-mass-shell dual amplitudes (II). Nucl. Phys. B72 (1974) 397.

[16] Y. Zhu. Vertex operator algebras, elliptic functions, and modular forms. 1990. 